\title{
Challenges and Opportunities in Content Distribution Networks: A Case Study
}

\author{
Muslim Elkotob \\ Media Broadcast, TDF Group \\ Berlin, Germany \\ muslim.elkotob@media-broadcast.com
}

\author{
Karl Andersson \\ Luleå tekniska universitet \\ Skellefteå, Sweden \\ karl.andersson@ltu.se
}

\begin{abstract}
In this paper we present some contemporary examples of research-oriented as well as commercial content delivery networks (CDN)s. We discuss how both types of CDNs complement each other in tackling the posed technical and commercial challenges. We also show which performance metrics such as quality of experience (QoE) and time to first byte (TTFB) best capture the dynamics of traffic and services in CDNs. The core of this paper is our proposed network architecture for CDN providers/operators. The architecture combines video multicast optimized trees and cross-layer coordination between the physical DWDM layer (L1) and network layer (L3) for achieving higher efficiency and lower latency values for live streaming and on demand (VoD) video. Due to the pilot implementation of the presented concept being limited in scale, we use simulations in order to perform proof-of-concept on a sufficiently large environment. Results show that there is a strong correlation between the TTFB and QoE metrics with the former taking on values as low as 75 msec in a national 3-tier network.
\end{abstract}

Keywordst: Content Delivery Network (CDN), Peering, IP Transit, Video Streaming, Quality of Experience.

\section{INTRODUCTION}

This article focuses on the technical challenges faced by a Content Delivery Network (CDN) provider in connection with innovative research-oriented ways to overcome them. Video streaming on its own poses performance challenges in IP networks due to the stringent requirements on performance parameters such as delay, jitter, etc. Furthermore, the burst-like pattern with which video traffic is generated causes the demand on bandwidth for video traffic to vary irregularly. A Content Delivery Network (CDN) provider has to cope with varying customer demands, assure acceptable quality of experience (QoE), and run a profitable business model.

We look at current CDN solutions of both formats academic (research oriented) and commercial. CDNs in the former category act as platforms for innovation in this area where as CDNs of the latter category have to deliver a high QoE) to a maximum number of customer devices with the available finite network resources. A commercial $\mathrm{CDN}$ is strongly governed by the underlying business model which tries to best capture the resources-revenues tradeoff. One of the major challenges for a content provider, service provider, or operator to launch a CDN is the initial investment for the basic infrastructure which includes the streaming servers with the content, the proxies, and the caching servers in addition to the network management software which governs the content exchange, fetching, trans-coding and streaming functionalities.
The research aspect in this paper deals mainly with proposing efficient ways to overcome the high cost hurdle and still maintain the required QoE level in a CDN by using innovative architectural adjustments and optimizing the service path (or meta-protocol) that governs the logic of CDN applications and services.

\section{STATE OF THE ART}

This section provides an overview of existing CDN models grouped in this paper into four categories: video streaming platforms, major business model types, commercial CDN operators, and academic CDNs.

\section{A. Video Streaming Platforms}

There are several well-known video streaming platforms which act as content delivery networks. They usually buy large portions of capacity in core and metro networks (nationwide and internationally) and they also operate content servers on which the multimedia CDN content is produced, stored, cached, or processed. Tier-1 ISPs (Internet Service Providers) are typical owners of such platforms.

Examples are Akamai [9], Cogent [10], and Level3 [11]. For those Tier-1 ISPs, the core business is event-based CDN video streaming. Upon the occurrence of events in the areas of sports, politics, or any other areas, those CDN providers are able to accommodate and serve intermediate providers and end-customers with multimedia content because their networks are provisioned and dimensioned accordingly.

Recent studies have shown that the nature of demand for multimedia content has changed in terms of the peak-toaverage data rate (PADR) by increasing from a factor of 2.9 to a factor of 6.5 according to a study conducted by the Swedish company Transmode [12]. For instance, a provider which has an average traffic load of $20 \mathrm{Gbps}$ in its backbone per segment averaged over the whole network has to dimension its backbone in such a way to accommodate 6.5 fold for the case when events are to be covered which is around $130 \mathrm{Gbps}$ in this case. This explains the trend for conglomerate and horizontal market growth trend based on expansion and acquisition pursued by Tier-1 ISPs. As the average traffic load grows, the peak traffic load to be covered for events in CDN mode is several fold of that capacity. Therefore, the asset or capital of each ISP that acts as a CDN is the peak capacity level it can accommodate. 


\section{B. Existing Business Models}

The most popular existing CDN business models include the pay-per-view or transaction-based model and the flat-rate or all-you-can-watch model. The way content is accessed and the interactions among stakeholders within a CDN eco-system determine the type of underlying business model. The two main types are content-centric CDNs and access-centric CDNs.

Content-centric CDNs are models where content providers pay $\mathrm{CDN}$ operators to accelerate their own content through the network to reach end-user devices with high QoE. QoE is the key here because it is seen as the guarantee of retaining customers as opposed to having them switch to providers whose video quality is believed and perceived to be better. So those CDNs are sort-of QoE guarantors from a customer view point. What CDNs in this category do is to employ routing intelligence to guide user requests to the local servers (e.g. CDN caching servers). Akamai [9] is an example of a contentcentric CDN. When multimedia content is successfully tagged and guided throughout the network in terms of request (signaling) and data flow, the chances of achieving a high level of QoE to the customer's satisfaction becomes higher. When the CDN provider pays co-location fees, it achieves its profit and break-even point from re-seller revenues. Figure 1 shows the basic architecture of a content-centric CDN system and the interactions within.

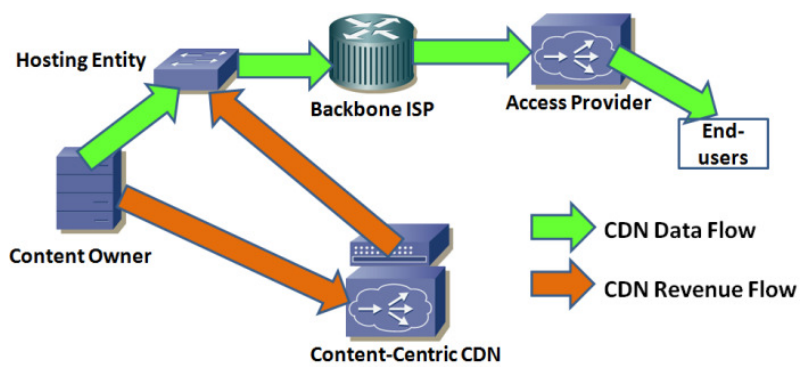

Figure 1: Content-Centric CDN Architecture and Flows.

The other type of CDN providers is the access-centric CDN type where the data flow for multimedia content is identical to its counter-case (the content-centric CDN model), but the revenue flow is from the access provider stakeholder towards the CDN provider. Access providers act as "fat-ISPs" where they provide not only access in terms of connectivity but also access to popular content delivered just as CDNs deliver their content. Subscribers of those fat-ISPs would then have access to popular multimedia content which is supplied by accesscentric $\mathrm{CDN}$ providers to their respective access providers.

\section{Commercial CDN Operators}

This is the category that forms the current and future market and there are several established players such as Netflix [1], Lovefilm [2], MyVideo [3], etc. What characterizes and differentiates commercial CDN operators are their size, their library of content, their mode of operation, and their positioning within their eco-system besides stakeholders in the underlying network architecture. Netflix for instance is known as one of the CDN giants in North America due to its large library of multimedia content and also its ability to serve customers nationwide with a broad range of content. As the volume of multimedia content in the library grows and as the demand for quality of experience (QoE) at high levels increases or at least stays stable, CDN operators are faced by the need to invest in their network infrastructure. This includes leased lines or dark fibers in the backbone, caching and streaming servers, intelligent content fetching mechanisms from the core network, and even hardware and software mechanisms for QoE provisioning and maintenance on the access links. Commercial CDN providers belong to either of the two business models presented in the previous subsection namely: content and access centric CDNs.

\section{Academic CDNs}

Academic CDNs are video streaming platforms and content delivery networks which offer caching and streaming services for video content within a research-like environment. Those CDNs allow for experimenting with the purpose of technically improving network performance in terms of QoS and QoE but they remain far from actually deployable networks for paying end-users within a commercially viable business model.

The most popular academic CDNs include: Globule (Vrije Universiteit Amsterdam) [4], FCAN (Flash Crowds Alleviation Network) [5], CoDeeN (Princeton University) [6], CoralCDN [6], and COMODIN (COoperative Media On-Demand on the InterNet) [8].

What academic CDNs have in common is their experimental nature for enhancing research in multimedia content distribution with quantitative measurable goals. Moreover, the deployment of experimental and research CDNs allows reaching architectural changes which provide significant performance boosts. This is necessary because the classical architectural models have started to reach their limitations and cannot cope with the growing volume of individual video stream (with the shift from SD to HD video) and collective volume as well. Several papers in the literature address this issue of the race between capacity growth and network architectural evolution. For instance in [13], Roy et al. introduce a metric called "network-cut exhaustion probability" and based on this parameter they determine upgrade requirements on the network. This constant methodological provisioning and dimensioning is an integral part of the CDN world as well. In [14], machine learning methods are proposed for classifying video traffic based on packet size in order to accommodate video streams in a better way and achieve acceptable performance (QoE) levels.

\section{MAJOR CHALLENGES FACING CDN OPERATORS}

In this section, we briefly cover the key challenges which a CDN operator or provider faces when serving, acquiring or retaining customers as well as in regard to the dynamics and competition faced on the CDN market.

Streaming video, whether in play-out or live mode composes the major part of CDN traffic. This traffic is packed into streams and sold as services as described in the previous section. Subscribing customers are either broadband connection subscribers at home or mobile device owners to whom streaming video is available. One barrier is the acquisition of mobile device (e.g. smart phone) customers to use CDN services; the main challenge here being the cost as 
well as QoE as discussed below. Achieving and maintaining high QoE levels with the growing traffic volumes and increasing QoS challenges in the core and access networks is a barrier to CDN operator success. Furthermore, the major CDN type which is content-centric as described in Section II in this paper faces a major cost barrier incurred by peering and IPtransit expenses.

\section{A. Barriers for the Mobile Video Market:}

Mobile video traffic is growing with different standards allowing for sufficient bandwidth and terminals capable of trans-coding and buffering as well as supporting a broad range of streaming video formats. However, CDN operators face the challenge of covering up for their costs and achieving profits when it comes to customers with mobile devices because the cost barrier for mobile connectivity is a problem in many countries. Mobile broadband, whether 3GPP LTE (Long Term Evolution) [15], or EDGE (Enhanced Data Rates for Global Evolution) [16], or any other wireless access technology capable of transporting video streams.

In [17], the authors propose variable-rate video coding to adapt to channel conditions and achieve higher bandwidth efficiency. Lowering the transmission rate to adapt to weaker QoS conditions could work within some bounds, but when watching live streaming videos or even playback from an archived multimedia CDN library, such techniques do reach their limitations. This issue has also been addressed in [18] for interactive video streaming where the performance bottlenecks where identified and partly alleviated. However in [18], various wireless technologies are used for higher bandwidth efficiency on their wireless devices for video streaming, whereas most business models involving CDN subscribers tie the user mostly to one access technology. Even when this is not the case, there has to be a default access technology, in mobile broadband mode in order to transport video streams the mobile device. The current price models where volume-based accounting is employed make CDN video services less attractive due to the price hurdle. On the other hand, the perceived quality or quality of experience (QoE) of video on small mobile terminals (e.g. smart phones) is not always sufficiently high enough to be adequate for $\mathrm{CDN}$ video streaming.

Operators try to achieve a balance between unicast and multicast streaming in their network in order to utilize resources (mainly bandwidth) as efficiently as possible and at the same time maintain performance levels for instance for retransmissions or adapting collective rates for a group of streams as demonstrated in [19]. Subscribers to flat-rate wireless broadband face the problem of service quality, since their traffic is not prioritized as "Premium" unless they pay additional charges and certain $\mathrm{CDN}$ providers also provide "Premium" content for additional charges. So watching premium content with premium quality (QoE) becomes an expensive venture for users and dims the growth chances of $\mathrm{CDN}$ providers on the mobile market. Ongoing research tries to address this challenge.
B. Achieving Sustainable Quality of Experience Levels

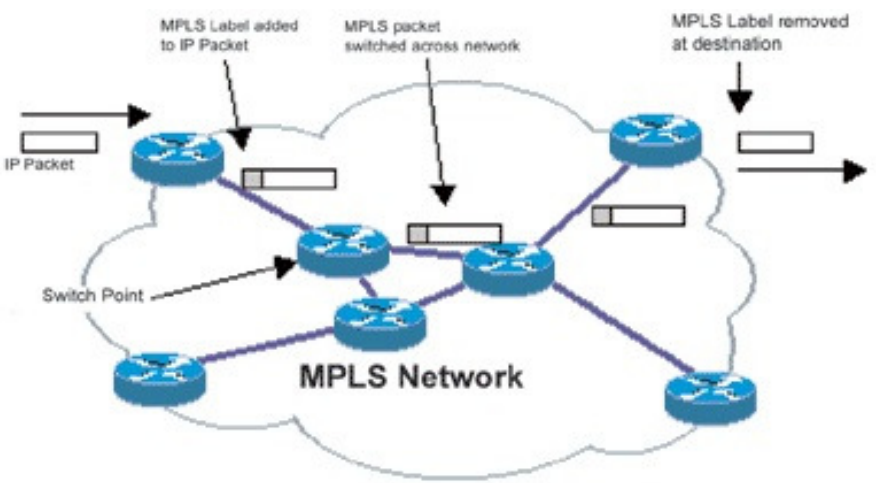

Figure 2: Multi-Protocol Label Switching in Backbone.

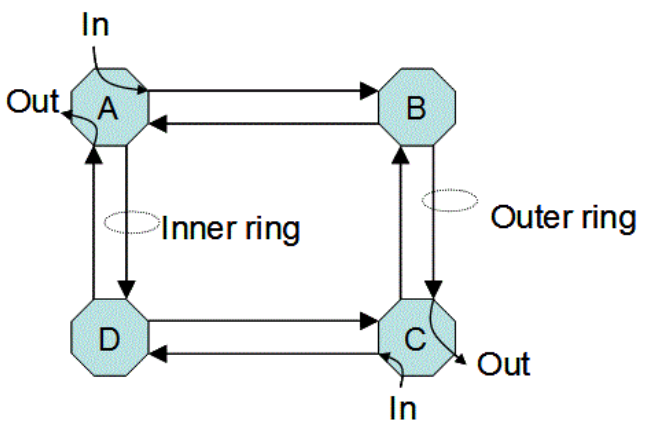

Figure 3: Ethernet Ring Protection in Backbone.

- $\quad$ MPLS (Figure 2): Most carriers and providers, including CDN providers try to maintain control over their own routing paths and thus deploy MPLS (Multi-Protocol Label Switching) and VPLS (Virtual Private LAN Services). When using either a Layer2 VPN service in the network or a L3 service-oriented MPLS service, scalability issues arise, especially when working with video traffic as in the case of CDN. The key to achieving high QoE in $\mathrm{CDN}$ networks. More details on this point are provided in Section V in this paper. Scalability issues arise because when using dynamic link metrics, as in line with the nature of CDN content can cause oscillations in the network and load the backbone links with the primary and backup paths almost to their limits [20]. On the other hand, MPLScompatible schemes such as Profile-Based Routing (PBR) work out fine for CDN networks when the load is relatively low or moderate, but with larger loads, problems occur too. The key point here is that IP routing as well as the MPLS path determination in the default as well as backup cases becomes contra-productive because of the complex routing relationships on intermediate nodes resulting from the video packet fetching from $\mathrm{CDN}$ caches and irregular user requests.

- Native Ethernet (Figure 3): As a response to the aforementioned challenges, we propose in this paper a cross-layer approach for $\mathrm{CDN}$ network operators and providers whereby $\mathrm{CDN}$ video traffic, whether multicast or unicast is packed into Native Ethernet Packets and transported over a L1 network consisting of leased lines or optical fibers (e.g. dark fiber, gray fiber) with the routing logic built into the protected wavelengths of the 
Reconfigurable Optical Add-Drop Multiplexers (ROADM)s in the optical network which are collocated with the backbone core network IP routers. This simplified design (with more information in Section V) allows bypassing many Layer3 core routers in the backbone and simply letting video streams exit at target locations, even in multicast mode using the EXMP (Ethernet Muxponder) technology where an example is available from [21]. The value-add is providing operators with connection-oriented transport services over native Ethernet based packetoptical networks, in this particular case for CDN operators.

\section{Peering and IP-Transit}

Both peering and IP-transit are key technical and monetary components in an operating CDN network. Those components can form hurdles to the launch or success of a CDN network or video platform because of the incurred costs are substantial in order to perform peering or IP-transit. The costs depend on the volume (e.g. committed, consumed, etc.) of multimedia traffic transferred or exchanged.

- Peering: Is when two ISPs or stakeholders make an agreement on a certain port to let the traffic destined to each of them pass through in order to reach its required destinations (end-users). Peering can be either public or private and it allows for packet exchange on a horizontal level (without crossing from a higher to a lower tier ISP or vice versa);

- IP Transit: Is the service where Internet traffic, and in this particular case CDN video streaming traffic, is transferred from one network to another, for further reach and connectivity. Seen on a larger scale, it is equivalent to having traffic of a small ISP or networked domain passed over to a Tier- 2 and then eventually to a Tier-1 ISP to reach the global platform.

After discussing the major challenges faced by CDN providers and highlighting their significance, we present in the next section a quick insight into opportunities in deploying a CDN network.

\section{OPPORTUNITIES FOR CDN NETWORKS}

For successful CDN network design, deployment and eventually profitable management, several factors have to come together, and the factors as well as their interactions are a result of ongoing research in this field. In this section, the most important metrics which characterize CDN-based services are outlined and also the organic growth model of CDN networks is introduced and discussed as a strength of such a model.

\section{A. Important CDN Metrics}

One important metric, also present in the web-server world is the so-called "Time To First Byte" or TTFB. This metric indicates the time it takes from the instant a request in a CDN network is initiated until the first CDN multimedia byte is play-ready on the user terminal (playout buffer). Due to the fact that video files have a varying range of length, and since routing over the core network could take place in multi-path mode for better load balancing and network utilization, defining a generic metric which reflects CDN video transport time is practically impossible. Therefore, as a benchmark and as a part of a Service Level Agreement (SLA) between a CDN provider and its customers, the TTFB is taken as an indicator.

Another important factor related to the video streams is the average packet size (or the content packet size if the stream is encoded that way). This parameter influences the behavior of the stream over the network and the scalability of QoS mechanisms employed on the individual links. In CDN networks, packet size and stream encoding and bundling are key features for link shaping and network performance modeling in both the access part and the core part. Resource modeling on the access link for accommodating many users and conforming to performance requirements is studied in a sample case study for vehicular networks with a $3 / 3.5 \mathrm{G}$ downlink (UMTS, EDGE [16]) in [23].

In Table 1 below, the metrics characterizing CDN models are summarized and explained.

\begin{tabular}{|l|l|l|}
\hline Metric & $\begin{array}{l}\text { Typical } \\
\text { Range }\end{array}$ & Remarks \\
\hline $\begin{array}{l}\text { Packet } \\
\text { Size }\end{array}$ & $\begin{array}{l}\text { 65-1518 } \\
\text { Bytes }\end{array}$ & $\begin{array}{l}\text { Very wide range; influences the } \\
\text { packets per second (PPS) frequency } \\
\text { upon limited capacity. Higher for I- } \\
\text { Frames and low for P-Frames and B- } \\
\text { Frames }\end{array}$ \\
\hline TTFB & $\begin{array}{l}50-350 \\
\text { msec }\end{array}$ & Time to First Byte: \\
\hline QoE MOS & $1-5$ & $\begin{array}{l}\text { Quality of Experience Mean Opinion } \\
\text { Score; theoretical range: 1-5; } \\
\text { practical range in this model 3.5-4.2 }\end{array}$ \\
\hline Load & $0-100 \%$ & $\begin{array}{l}\text { It is possible to load the network with } \\
\text { more than 100\% of its link capacity }\end{array}$ \\
\hline Utilization & $0-1.0$ & $\begin{array}{l}\text { It reflects the effective usage of link } \\
\text { capacity in terms of goodput as } \\
\text { opposed to the more generic metric } \\
\text { reflecting the load on the network }\end{array}$ \\
\hline
\end{tabular}

Table 1: Important CDN Metrics.

\section{B. Organic CDN Growth}

Because CDN is the area where content management and QoS mechanisms on a closed domain network meet, the organic growth of such networks is possible. Stakeholders can interact and expand or share responsibility in way which allows expansion. For instance, an infrastructure provider (or operator) can open its network to a content provider to run its content and then both can expand in terms of content and coverage depending on what it is most beneficial to acquire.

The strict distinction between stakeholders is disappearing, and this lowers the price/cost barrier and allows for healthy expansion of CDN networks. For this reason, when establishing a CDN network, the assumption of linear-scale organic growth can be made as a basis for profit projection. 


\section{Proposed Architecture AND Model Evaluation}

We propose in this paper synchronizing the play-out centers which are the CDN multimedia content sources via broadband links such as 10Gbps leased lines (LL) or dedicated wavelengths on a dark fiber. Then the selection of Points of Presence (PoPs) among existing backbone node locations has to take place during network design while focusing on the following factors:

- Geographical factors: e.g. geo-blocking, distance from play-out centers, distribution and density of CDN users, etc.;

- Economic/Business factors: e.g. peeing opportunities with last-mile carriers, access to end-customers via peering/IP-transit, etc.

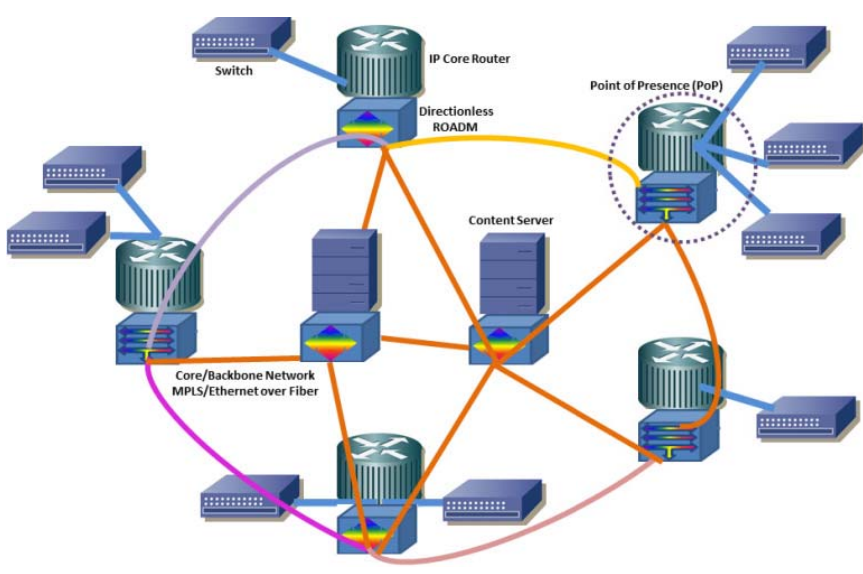

Figure 4: Proposed CDN Architecture.

Most operators who own or run a leased backbone deploy MPLS (Multi-Protocol Label Switching) which combines Layer-2 and Layer-3 features with one or two alternative paths for traffic re-routing upon the failure of the default multi-hop path between two nodes.

The issue with MPLS is that it does not scale well with larger bandwidths and increasing loads in the network. With Standard Definition (SD) video traffic with streams in the order of several $100 \mathrm{Mbps}$ data rates, MPLS could still scale fine in the backbone domain. However, with currently available High Definition (HD) streams with a bandwidth ranging between 1.6 and $3 \mathrm{Gbps}$, MPLS does not scale that well anymore; moreover, when using video in multicast mode, scalability becomes increasingly low. Palmieri et al. [25] provide a detailed performance analysis of MPLS networks with focus on QoS-related aspects. Optical-speed media streaming is a focus issue in [25], and Content Delivery Networking (CDN), which is the topic of this paper, is a specific case of high-bandwidth streaming networking over an IP and (or) optical backbone from the content source towards the edges.

To evaluate the benefits of the proposed architecture, we built a simulation model using OPNET IT Guru [24] for the topology in Figure 4 with two play-out centers as sources of multimedia content and five points of presence (PoPs) where multimedia content is fetched from the source and cached besides being streamed into the last mile or access network segment. The data path shown in Figure 1 is the same as the one used in the simulation model. All connections whether between PoPs or play-out centers are 10 Gbps leased lines in bundles of 3 per segment capable of supporting the capacity (with overhead) of 30 Gbps per link. High Definition (HD) streams of video content are used to fill up the capacity of the links uniformly with discrete values of 30, 50, 70, 90 and 110 percent in both modes video on demand (VoD) and Live Streaming. For Live Streaming, short-tail traffic is used whereas for VoD, long-tail traffic is used in the simulation. The video packet size is kept at the maximum of 1518 bytes and the packet rate is throttled and adjusted so as to fill the capacity with the different line rates on the $\mathrm{x}$-axis of the performance graphs (namely 30\%, 50\%, 70\%, 90\%, and $110 \%$ of the $30 \mathrm{Gbps}$ capacity of the links). On the backbone, two alternatives on top of the optical (L1) equipment are used for performance comparison: Native Ethernet in Ethernet Protected Ring (EPR) mode and Multi-Protocol Label Switching. The main difference between those two underlying backbone mechanisms is that the former uses ITU-T G.8032 automatic protection switching by changing the transport direction on the ring upon a break or failure, whereas the latter uses one or two alternative switched-label paths to the default route upon breaks or extreme load.

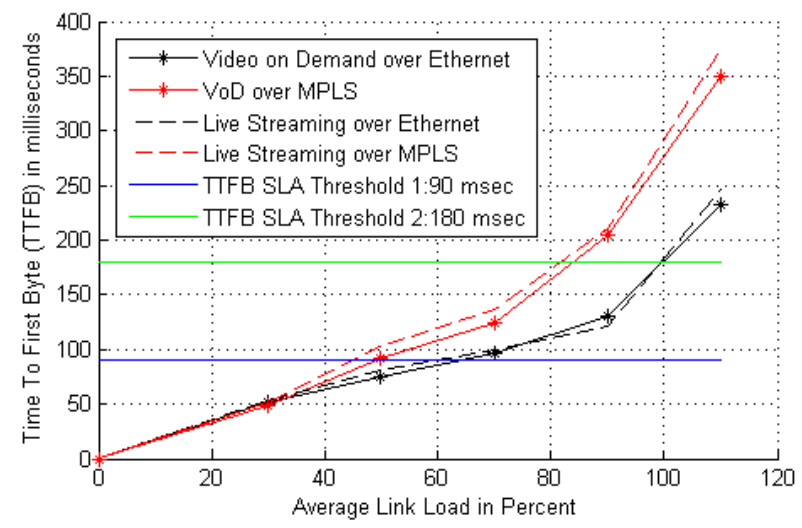

Figure 5: CDN TTFB Performance Ethernet vs. MPLS.

As Figure 5 shows, for relatively low loads, EPR and MPLS perform comparably for CDN video traffic in terms of TTFB (the time it takes to put the first video byte or packet on the player of the requesting client). However, for moderate loads of around 50\%, EPR outperforms MPLS with 75 milliseconds TTFB times whereas MPLS delivers a TTFB of approximately $90 \mathrm{msec}$. For typical Service Level Agreement (SLA) bounds of 90 and $180 \mathrm{msec}$ for premium and regular levels respectively, the EPR alternative for both $\mathrm{VoD}$ and live streaming remains within the acceptable interval even for higher loads as compared to MPLS which exits the acceptable SLA level already for a load of $80 \%$ whereas EPR does that for a loads larger than $92 \%$.

For QoE modeling, the Mean Opinion Score for video is used; this metric is explained in Table 1 and some QoE example models for video are provided in [22]. For this model QoE is taken as the linear weighted sum of TTFB, delay 
bound, and packet loss rate (PLR) with all coefficients being equal and the result normalized to fit the MOS scale of 1-5. Both backbone technologies (ERP and MPLS) degrade in terms of QoE as the load is increased, however, due to its simpler operating mode, ERP degrades more slowly in MOS value and is thus able to deliver better perceived quality of CDN video even with loads in the range of $70-80 \%$. An acceptable QoE MOS value is normally not lower than 3.63.7. An MPLS network drops below that QoE level and ceases to scale well with HD video streams already at loads around $60 \%$ whereas ERP maintains acceptable QoE values for loads up to almost $80 \%$ as observed in Figure 6.

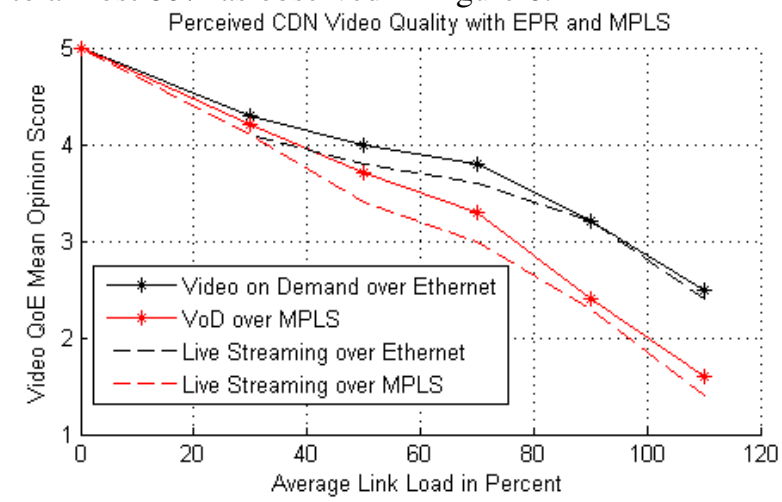

Figure 6: Quality of Experience in CDN with ERP and MPLS.

\section{CONCLUSION AND FUTURE WORK}

In this paper we have presented an overview of existing CDN technologies and platforms and identified a current trend which has started to reach its limits when relying on a L2 and L3 based solution using MPLS in the backbone. Moreover, using a cross-layer L1-L3 architecture and native Ethernet for video transport and broadcasting over the backbone delivers a better performance as simulation results show. The time to first byte (TTFB) metric used for web server performance also proves to be a viable metric for performance benchmarking in the CDN domain. The paradigm conveyed by this paper is a performance QoE (quality of experience) driven architectural design for getting more out of the network and sustaining growth and end-user satisfaction. Such an approach, once proven successful could become a trend-setter for further research in the area of multimedia content distribution and CDN networks.

\section{ACKNOWLEDGMENT}

We would like to thank all project partners including CDN providers, Tier-1 ISPs, and other types of stakeholders. The collaboration with those stakeholders has pushed our CDN research forward and opened up new frontiers.

\section{REFERENCES}

[1] Netflix CDN: www.netflix.com; accessed 12.06.2012

[2] Lovefilm CDN: www.lovefilm.com; accessed 12.06.2012

[3] MyVideo CDN: www.myvideo.de; accessed on 12.06 .2012

[4] Globule research CDN: www.globule.org; accessed on 12.06.2012
[5] C. Pan, M. Atajanov, M. Hossain, T. Shimokawa, and N. Yoshida: FCAN: Flash Crowds Alleviation Network; in proceedings of ACM SAC'06, April 23-27, 2006, Dijon, France

[6] CoDeeN research CDN for PlanetLab: http://codeen.cs.princeton.edu/; accessed on 12.06.2012

[7] Coral Content Distribution Network research CDN: www.coralcdn.org; accessed on 12.06.2012

[8] G. Fortino, C. Palau, W. Russo, and M. Esteve; The COMODIN System: A CDN-based Platform for Cooperative Media On-Demand on the Internet; in proceedings of the $10^{\text {th }}$ International Conference on Distributed Multimedia Systems (DMS'04)

[9] Akamai CDN provider: www.akamai.com, accessed on 15.06.2012

[10] Cogent CDN provider: www.cogent.com, accessed on 15.06.2012

[11] Level3 CDN provider: www.level3.com, accessed on 15.06.2012

[12] Transmode: www.transmode.com, accessed on 14.06.2012

[13] R. Roy, "Managing Traffic Growth in Telecom Mesh Networks", in Proceedings of the $17^{\text {th }}$ International Conference on Computer and Communication Networks ICCCN'08, Davis, CA, USA 2008

[14] K. Takeshita, T. Kurosawa, M. Tsujino, M. Iwashita, M. Ichino, and N. Komatsu, "Evaluation of HTTP Video Classification Methods Using Flow Group Information", In Proceedings of the $14^{\text {th }}$ International Telecommunications Network Strategy Symposium 2010, Warsaw, Poland, 23-27 September 2010

[15] 3GPP ( $3^{\text {rd }}$ Generation Partnership Project) Long Term Evolution (LTE): www.3gpp.org/LTE, accessed 10.06.2012

[16] 3GPP EDGE (Enhanced Data rates for Global Evolution): www.3gpp.org/specifications, accessed 09.06.2012

[17] L. Al-Jobouri, M. Fleury, and M. Ghanbari, "Bandwidth Efficient DataPartitioned Video Streaming for Broadband Wireless", in proceedings of the $18^{\text {th }}$ International Conference on Signals, Systems, and Image Processing IWSSIP 2011, Colechester, UK, 16-18 June 2011

[18] K. Andersson, D. Granlund, M. Elkotob, and C. Åhlund, "Bandwidth Efficient Mobility Management in Heterogeneous Wireless Networks", in Proceedings of the 7th IEEE Consumer Communications and Networking Conference CCNC 2010, Pages 508-513, Las Vegas NV, USA, January 2010

[19] L. Al-Jobouri, M. Fleury, and M. Ghanbari, "Multicast and Unicast Video Streaming with Rateless Channel-Coding over Broadband Wireless", In Proceedings of Consumer Communications and Networking Conference CCNC 2012, pp 737-741, Las Vegas, NV, USA January 2012

[20] S. Yilmaz and I. Matta, "On the Scalability-Performance Tradeoffs in MPLS and IP Routing", In Proceedings of the SPIE ITCOM'2002: Scalability and Traffic Control in IP Networks, 2002

[21] Ethernet Muxponder (EMXP) Technology example: http://www.transmode.com/press-releases/transmode-brings-mpls-tp-topacket-optical-metro-networking

[22] M. Elkotob, D. Granlund, K. Andersson, and C. Ahlund, "Multimedia QoE Optimized Management using Prediction and Statistical Learning", In Proceedings of the IEEE $35^{\text {th }}$ Conference on Local Computer Networks IEEE LCN 2010 pp 328-331, Denver, CO, USA, October 2010

[23] M. Elkotob, "Architectural, Service and Performance Modeling for an IMS-MBMS-based Application", in Proceedings of the IEEE International Conference on Communications (IEEE ICC) 2010, Cape Town, South Africa, May 2010

[24] OPNET IT Guru Academic Edition Simulation Environment: http://www.opnet.com/university_program/itguru_academic_edition

[25] F. Palmieri. "MPLS DiffServ-Enabled Traffic Engineering: A Scalable QoS Model for Optical-Speed Media Streaming Networks". In: High Speed Networks and Multimedia Communications. Ed. by Zoubir Mammeri and Pascal Lorenz. Lecture Notes in Computer Science, Vol. 3079. Springer Berlin / Heidelberg, 2004, pp. 301-313. 